\title{
Quasi-equilibrium lattice Boltzmann method
}

\section{Journal Article}

\section{Author(s):}

Ansumali, S.; Arcidiacono, S.; Chikatamarla, S. S.; Prasianakis, N. I.; Gorban, A. N.; Karlin, llya

Publication date:

2007

Permanent link:

https://doi.org/10.3929/ethz-b-000005815

Rights / license:

In Copyright - Non-Commercial Use Permitted

\section{Originally published in:}

The European Physical Journal B 56(2), https://doi.org/10.1140/epjb/e2007-00100-1 


\title{
Quasi-equilibrium lattice Boltzmann method
}

\author{
S. Ansumali ${ }^{1}$, S. Arcidiacono ${ }^{2}$, S.S. Chikatamarla ${ }^{3}$, N.I. Prasianakis $^{3}$, A.N. Gorban ${ }^{4}$, and I.V. Karlin ${ }^{3, \text { a }}$ \\ 1 CBE, Nanyang Technological University, 639798 Singapore, Singapore \\ 2 Paul Scherrer Institute, Combustion Research Unit, 5232 Villigen PSI, Switzerland \\ 3 Aerothermochemistry and Combustion Systems Lab, ETH Zurich, 8092 Zurich, Switzerland \\ 4 Department of Mathematics, University of Leicester, Leicester LE1 7RH, UK
}

Received 29 September 2006 / Received in final form 2 February 2007

Published online 12 April 2007 - (C) EDP Sciences, Società Italiana di Fisica, Springer-Verlag 2007

\begin{abstract}
A general lattice Boltzmann method for simulation of fluids with tailored transport coefficients is presented. It is based on the recently introduced quasi-equilibrium kinetic models, and a general lattice Boltzmann implementation is developed. Lattice Boltzmann models for isothermal binary mixtures with a given Schmidt number, and for a weakly compressible flow with a given Prandtl number are derived and validated.
\end{abstract}

PACS. 47.11.-j Computational methods in fluid dynamics - 05.20.Dd Kinetic theory

\section{Introduction}

The lattice Boltzmann (LB) method is a powerful approach to hydrodynamics, with applications ranging from large Reynolds number flows to flows at a micron scale, porous media and multi-phase flows [1]. However, in spite of its rapid development over a decade, the method is still lacking a systematic and flexible construction of numerical schemes for situations beyond a simple fluid, such as mixture models with a given Schmidt number, or thermal models with a given Prandtl number.

In this paper, we introduce a general method of constructing the lattice Boltzmann models. Our approach is based on recently introduced quasi-equilibrium (QE) kinetic models $[2,3]$. The structure of the paper is as follows: first, for the sake of completeness, we remind the construction of continuous time-space QE models. After that, we derive a lattice Boltzmann discretization scheme of the QE models. The resulting quasi-equilibrium lattice Boltzmann method (QELBM) is illustrated with two examples of particular interest. The first example is the thermal model with a prescribed Prandtl number. The second example is the isothermal binary mixture model with a prescribed Schmidt number. Basic steps of construction of these models are presented and numerical validation is provided.

\footnotetext{
a e-mail: karlin@lav.mavt.ethz.ch
}

\section{Quasi-equilibrium kinetic models}

\subsection{General}

We address the class of lattice Boltzmann models equipped with the Boltzmann entropy function of the form,

$$
H=\sum_{i=1}^{n} f_{i} \ln \left(\frac{f_{i}}{W_{i}}\right),
$$

where $f_{i} \geq 0$ are populations of the discrete velocities $v_{i}$, $i=1, \ldots, n$, and $W_{i}>0$ are corresponding weights. A wide class of relevant entropy functions (1) pertinent to simulation of hydrodynamics was described in [4-8]. In the examples below we shall use the $H$-function for the isothermal model [4] and the recently introduced weakly compressible thermal model [6] in two dimensions, for which the set of nine velocities $v_{i}$ and corresponding weights $W_{i}$ are

$$
\begin{aligned}
v_{x} & =\{0,1,0,-1,0,+1,-1,-1,+1\} \\
v_{y} & =\{0,0,1,0,-1,+1,+1,-1,-1\} \\
W & =(1 / 36)\{16,4,4,4,4,1,1,1,1\} .
\end{aligned}
$$

We begin with a generic construction of the quasiequilibrium kinetic models $[2,3]$ specified for the discrete velocity case. Let $M=\left\{M_{1}, \ldots, M_{k_{M}}\right\}$ be a set of locally conserved fields, and $N=\left\{N_{1}, \ldots, N_{k_{N}}\right\}$ be a set of quasi-conserved slow fields. Functions $M_{m}$ and $N_{l}$ are assumed linear functions (moments) of the populations, 
$M_{m}=\sum_{i} M_{m i} f_{i}$ and $N_{l}=\sum_{i} N_{l i} f_{i}$. The choice of $M$ and $N$ depends on the particular problem but we always assume that the density is included into the list $M$, $\rho=M_{1}=\sum_{i=1}^{n} f_{i}$. The equilibrium population vector $f^{\mathrm{eq}}(M)$ is defined as the minimizer of the $H$-function (1) under fixed $M$. The quasi-equilibrium $f^{*}(M, N)$ is defined as the minimizer of $H$ under fixed $M$ and $N$. By construction, functions $f^{*}$ and $f^{\text {eq }}$ satisfy consistency relations

$$
\begin{aligned}
M\left(f^{*}(M, N)\right) & =M, \\
N\left(f^{*}(M, N)\right) & =N, \\
M\left(f^{\mathrm{eq}}(M)\right) & =M .
\end{aligned}
$$

The quasi-equilibrium kinetic model reads

$$
\begin{aligned}
\partial_{t} f_{i}+v_{i \alpha} \partial_{\alpha} f_{i} & =-\frac{1}{\tau_{1}}\left[f_{i}-f_{i}^{*}(M(f), N(f))\right] \\
& -\frac{1}{\tau_{2}}\left[f_{i}^{*}(M(f), N(f))-f_{i}^{\mathrm{eq}}(M(f))\right] .
\end{aligned}
$$

Denoting the right hand side (the collision integral) as $Q_{i}$, it is easy to see that consistency condition implies local conservation laws, $M(Q)=0$. Note that the first part of the collision integral which describes relaxation to the quasi-equilibrium, also conserves the $N$-fields, $N\left(f-f^{*}\right)=$ 0 . Moreover, it is straightforward to prove the $H$-theorem. For that, it suffices to rewrite

$$
Q_{i}=-\frac{1}{\tau_{2}}\left[f_{i}-f_{i}^{\mathrm{eq}}\right]-\left(\frac{\tau_{2}-\tau_{1}}{\tau_{1} \tau_{2}}\right)\left[f_{i}-f_{i}^{*}\right] .
$$

The entropy production $\sigma=\sum_{i=1}^{n}\left(\partial H / \partial f_{i}\right) Q_{i}$ becomes

$$
\begin{aligned}
\sigma= & -\frac{1}{\tau_{2}} \sum_{i=1}^{n} \ln \left(\frac{f_{i}}{f_{i}^{\mathrm{eq}}}\right)\left(f_{i}-f_{i}^{\mathrm{eq}}\right) \\
& -\left(\frac{\tau_{2}-\tau_{1}}{\tau_{1} \tau_{2}}\right) \sum_{i=1}^{n} \ln \left(\frac{f_{i}}{f_{i}^{*}}\right)\left(f_{i}-f_{i}^{*}\right),
\end{aligned}
$$

and is non-positive semi-definite provided relaxation times satisfy the hierarchy,

$$
\tau_{1} \leq \tau_{2} .
$$

Thus, in the QE models, relaxation to the equilibrium is split in two steps. In the first step, the distribution functions relaxes to the quasi-equilibrium with the faster relaxation time $\tau_{1}$. In the second step, the quasi-equilibrium relaxes to the equilibrium with the slower relaxation times $\tau_{2}$. If $\tau_{1}=\tau_{2}$, the intermediate relaxation step to the quasi-equilibrium disappears from (4), and it reduces to the Bhatnagar-Gross-Krook (BGK) model.

\subsection{Triangle entropy method}

For a practical implementation, explicit form of the functions $f_{i}^{\text {eq }}(M)$ and $f_{i}^{*}(M, N)$ are required. While for most of the cases, the equilibrium $f_{i}^{\text {eq }}$ can be found explicitly either in a closed form or in a form of expansion, explicit construction of the quasi-equilibrium is case-dependent. Here we suggest a simple way to find quasi-equilibria in explicit form by perturbation around the equilibrium $[2,9]$.
Let us assume that the equilibrium $f^{\mathrm{eq}}(M)$ has been found explicitly, and that near the equilibrium

$$
f^{*}=f^{\mathrm{eq}}(M)+\delta f^{*}(M, N) .
$$

Expanding the entropy function $H(1)$ at the equilibrium to quadratic terms, we obtain

$$
\begin{aligned}
H\left(f^{\mathrm{eq}}+\delta f\right)= & H\left(f^{\mathrm{eq}}\right)+\sum_{i=1}^{n} \delta f_{i}\left(\ln \left(\frac{f_{i}^{\mathrm{eq}}}{W_{i}}\right)+1\right) \\
& +\frac{1}{2} \sum_{i=1}^{n} \frac{\delta f_{i}^{2}}{f_{i}^{\mathrm{eq}}}+O\left(\delta f^{3}\right) .
\end{aligned}
$$

The quadratic expansion of the entropy function (9) maintains convexity, and we find its minimizer $\delta f^{*}$ subject to the linear constraints

$$
\begin{aligned}
& \sum_{i=1}^{n} M_{k i} \delta f_{i}^{*}=0, \\
& \sum_{i=1}^{n} N_{l i} \delta f_{i}^{*}=N_{l}-N_{l}^{\mathrm{eq}},
\end{aligned}
$$

where $N^{\mathrm{eq}}=N\left(f^{\mathrm{eq}}(M)\right)$ are the values of the nonconserved fields at equilibrium. Solution to this minimization problem has the form,

$$
\begin{aligned}
f^{*} & =f_{i}^{\mathrm{eq}}(M)+\delta f_{i}^{*} \\
& =f_{i}^{\mathrm{eq}}(M)\left(1+\sum_{s=1}^{k_{M}} \lambda_{s} M_{s i}+\sum_{s=1}^{k_{N}} \chi_{s} N_{s i}\right),
\end{aligned}
$$

where Lagrange multipliers $\lambda_{s}$ and $\chi_{s}$ are found upon substituting function (11) into the constrains (10), and solving the $\left(k_{M}+k_{N}\right) \times\left(k_{M}+k_{N}\right)$ linear algebraic problem. Introducing a $\left(k_{M}+k_{N}\right)$-dimensional vector of Lagrange multipliers, $(\lambda, \chi)=\left(\lambda_{1}, \ldots, \lambda_{k_{M}}, \chi_{1}, \ldots, \chi_{k_{N}}\right)$, and matrices

$$
\begin{aligned}
\left(A_{M M}\right)_{k l} & =\sum_{i=1}^{n} M_{k i} f_{i}^{\mathrm{eq}} M_{l i}, k, l=1, \ldots, k_{M} \\
\left(A_{M N}\right)_{k l} & =\sum_{i=1}^{n} M_{k i} f_{i}^{\mathrm{eq}} N_{l i}, k=1, \ldots, k_{M}, l=1, \ldots, k_{N} \\
\left(A_{N N}\right)_{k l} & =\sum_{i=1}^{n} N_{k i} f_{i}^{\mathrm{eq}} N_{l i}, k, l=1, \ldots, k_{N}
\end{aligned}
$$

we find the solution in the matrix form,

$$
\left(\begin{array}{c}
\lambda \\
\chi
\end{array}\right)=\left(\begin{array}{ll}
A_{M M} & A_{M N} \\
A_{M N}^{T} & A_{N N}
\end{array}\right)^{-1}\left(\begin{array}{c}
0 \\
N-N^{\mathrm{eq}}
\end{array}\right),
$$

where $T$ denotes transposition, and $N-N^{\text {eq }}$ is the $k_{N}$-dimensional vector $\left(N_{1}-N_{1}^{\mathrm{eq}}, \ldots, N_{k_{N}}-N_{k_{N}}^{\mathrm{eq}}\right)$. Note that the solution depends linearly on the deviation of the non-conserved fields $N-N^{\mathrm{eq}}$ and non-linearly on the locally conserved $M$. For this quasi-linear quasi-equilibrium, 
the entropy production becomes

$$
\begin{aligned}
\sigma= & -\frac{1}{\tau_{2}} \sum_{i=1}^{n} \frac{\left(f_{i}-f_{i}^{\mathrm{eq}}\right)^{2}}{f_{i}^{\mathrm{eq}}} \\
& -\left(\frac{\tau_{2}-\tau_{1}}{\tau_{1} \tau_{2}}\right) \sum_{i=1}^{n} \frac{\left(f_{i}-f_{i}^{*}\right)^{2}}{f_{i}^{\mathrm{eq}}}+O\left(\delta f^{3}\right) .
\end{aligned}
$$

Thus, with the use of the triangle entropy method, the kinetic model satisfies the entropy production inequality (both of the two quadratic forms in (14) are non-positive semi-definite) once the populations remain close to the local equilibrium. This is sufficient for most of the applications.

\section{Quasi-equilibrium lattice Boltzmann method}

We shall now derive a second-order time discretization for the generic kinetic equation (4). Since the derivation only uses the consistency condition (3), it is equally applicable to exact quasi-equilibria and to those obtained by the triangle entropy method (8). Following [10], kinetic equations (4) are integrated in time from $t$ to $t+\delta t$ along characteristics, and the time integral of the right hand side is evaluated by trapezoidal rule. Introducing a map

$$
f_{i} \rightarrow g_{i}=f_{i}-\frac{\delta t}{2} Q_{i}(f),
$$

the result is written as

$$
\begin{aligned}
g_{i}\left(x+c_{i} \delta t, t+\delta t\right)= & g_{i}(x, t)-\omega_{1}\left[g_{i}(x, t)-f_{i}^{*}(x, t)\right] \\
& -\frac{\omega_{1} \tau_{1}}{\tau_{2}}\left[f_{i}^{*}(x, t)-f_{i}^{\mathrm{eq}}(x, t)\right],
\end{aligned}
$$

where

$$
\begin{aligned}
\omega_{1} & =\frac{2 \delta t}{2 \tau_{1}+\delta t}, \\
f^{*}(x, t) & =f^{*}(M(f), N(f)), \\
f_{i}^{\mathrm{eq}}(x, t) & =f_{i}^{\mathrm{eq}}(M(f)) .
\end{aligned}
$$

Note that the right hand side in (16) still contains terms which depend on populations $f$ rather than on the function $g$ (15). In order to obtain a closed-form equation solely in terms of functions $g$, we first remark that, taking locally conserved moments of the map (15) we have (same as in the BGK case) $M(f)=M(g)$, thus $f^{\mathrm{eq}}(f)=f^{\mathrm{eq}}(g)$ in the second term in (16). For the quasi-conserved fields $N$, the situation is slightly different. Evaluating the moments $N$ of the map (15), we obtain

$$
N(g)=N(f)+\frac{\delta t}{2 \tau_{2}}\left(N(f)-N^{\mathrm{eq}}(f)\right) .
$$

Inverting this relation, and substituting it into (16), after simple transformations we obtain:

$$
\begin{aligned}
g_{i}\left(x+c_{i} \delta t, t+\delta t\right)= & \left(1-\omega_{1}\right) g_{i}+\omega_{1}\left(\frac{\tau_{1}}{\tau_{2}}\right) f_{i}^{\mathrm{eq}}(M) \\
& +\omega_{1}\left(\frac{\tau_{2}-\tau_{1}}{\tau_{2}}\right) f_{i}^{*}\left(M, N^{\prime}\right),
\end{aligned}
$$

where $M=M(g)$, while $N^{\prime}$ is evaluated according to the rule

$$
N^{\prime}=\left(1-\frac{\delta t}{2 \tau_{2}+\delta t}\right) N(g)+\frac{\delta t}{2 \tau_{2}+\delta t} N^{\mathrm{eq}}(g) .
$$

Equation (19) is the basic second-order time stepping algorithm for the quasi-equilibrium lattice Boltzmann models, and is the main result of this paper. It is important to note the shift in the dependence of the quasi-equilibrium population $f^{*}$, it does not depend just on $N(g)$ but rather on a convex linear combination between $N(g)$ and the equilibrium value $N^{\mathrm{eq}}(g)$. Discretization in space depends on the problem at hand (in the simplest case, the lattice Boltzmann discretization is readily applicable). We shall now proceed with specific examples of QELBM.

\section{Examples}

\subsection{One-component fluid with a given Prandtl number}

Navier-Stokes equations for a one-component compressible fluid are characterized by the Prandtl number, $\mathrm{Pr}=$ $\left(C_{p} \mu\right) / \kappa$, where $C_{p}$ is the specific heat at constant pressure $\left(C_{p}=(D+2) / 2\right.$ is specific heat of ideal gas below $), \mu$ is viscosity coefficient, and $\kappa$ is thermal conductivity. When a single relaxation time kinetic equation is used (for example, the BGK model), this results in a fixed Prandtl number. As we shall see it below, physical consistency of the QE models, the relaxation time hierarchy (7), implies that two models with two different quasi-equilibria are required in order to cover the entire range of Prandtl number. For the sake of concreteness, we consider a simple, weakly compressible flow model on the standard twodimensional $D 2 Q 9$ lattice $[6,13]$. The locally conserved fields $M$ are density $\rho$, momentum density $\boldsymbol{j}$, and pressure density $p$,

$$
\sum_{i=1}^{n}\left\{1, \boldsymbol{v}_{i}, v_{i}^{2}\right\} f_{i}=\left\{\rho, \boldsymbol{j}, 2 p+\rho^{-1} j^{2}\right\} .
$$

To second order in the momentum, the equilibrium reads $[6,13]$ :

$$
\begin{array}{r}
f_{i}^{\mathrm{eq}}(\rho, \boldsymbol{j}, p)=\rho\left(1-\frac{p}{\rho}\right)^{2}\left(\frac{\frac{p}{\rho}}{2\left(1-\frac{p}{\rho}\right)}\right)^{v_{i}^{2}}\left[1+\frac{v_{i \alpha} j_{\alpha}}{p}\right. \\
\left.+\frac{j_{\alpha} j_{\beta}}{2 p^{2}}\left(v_{i \alpha} v_{i \beta}-\frac{4\left(\frac{p}{\rho}\right)^{2}+v_{i}^{2}\left(1-3 \frac{p}{\rho}\right)}{2\left(1-\frac{p}{\rho}\right)} \delta_{\alpha \beta}\right)\right] .
\end{array}
$$

This model operates in a small temperature window $T=$ $p / \rho$, around the reference temperature $T_{0}=1 / 3$ (see Refs. $[6,13]$ for an estimate of the temperature window). We remark that, due to a low symmetry of the velocity set, the lattice BGK (LBGK) model with the equilibrium (22) gives $\operatorname{Pr}_{\mathrm{LBGK}}=4$ [13] (not $\operatorname{Pr}=1$, as in the continuous kinetic theory or thermal lattice Boltzmann models with a higher symmetry $[5,8])$. In the first case $\left(\operatorname{Pr}<\operatorname{Pr}_{\text {LBGK }}\right)$, 
the specified slow variables $N$ are the components of the heat flux $\boldsymbol{q}$,

$$
q_{\alpha}=\sum_{i=1}^{n}\left(v_{i \alpha}-u_{\alpha}\right)\left(\boldsymbol{v}_{i}-\boldsymbol{u}\right)^{2} f_{i}
$$

where $\boldsymbol{u}=\boldsymbol{j} / \rho$ is the mean velocity. In the opposite case $\left(\operatorname{Pr}>\operatorname{Pr}_{\text {LBGK }}\right)$, the slow variables $N$ are the components of the stress tensor $\Theta$

$$
\Theta_{\alpha \beta}=\sum_{i=1}^{n}\left[\left(v_{i \alpha}-u_{\alpha}\right)\left(v_{i \beta}-u_{\beta}\right)-\frac{2}{D} \delta_{\alpha \beta}\left(\boldsymbol{v}_{i}-\boldsymbol{u}\right)^{2}\right] f_{i} .
$$

Explicit expressions for the pertinent quasi-equilibria, $f_{i}^{*}(\rho, \boldsymbol{j}, p, \boldsymbol{q})$ (case $\left.(23), \operatorname{Pr} \leq \operatorname{Pr}_{\mathrm{LBGK}}\right)$ and $f_{i}^{*}(\rho, \boldsymbol{j}, p, \boldsymbol{\Theta})$ (case $(24), \operatorname{Pr} \geq \operatorname{Pr}_{\text {LBGK }}$ ) are easily found using the explicit formulas of the triangle entropy method, equations (11) and (13), and are not displayed here. Using the Chapman-Enskog method, we derive the hydrodynamic equations (Navier-Stokes-Fourier) for the density, momentum and temperature from the continuous QE models (4) with the corresponding quasi-equilibria $f_{i}^{*}(\rho, \boldsymbol{j}, p, \boldsymbol{q})$ and $f_{i}^{*}(\rho, \boldsymbol{j}, p, \boldsymbol{\Theta})$ (note that the explicit form of $f_{i}^{*}$ is not required to perform this analytical computation, the consistency condition 3 suffices). The viscosity $\mu$ and the thermal conductivity $\kappa$ coefficients thus obtained, imply the following Prandtl number:

$$
\begin{aligned}
& \mu=\rho T_{0} \tau_{1}, \kappa=\frac{1}{2} \rho T_{0} \tau_{2} \Rightarrow \operatorname{Pr}=4 \frac{\tau_{1}}{\tau_{2}} \leq \operatorname{Pr}_{\mathrm{LBGK}} \\
& \mu=\rho T_{0} \tau_{2}, \kappa=\frac{1}{2} \rho T_{0} \tau_{1} \Rightarrow \operatorname{Pr}=4 \frac{\tau_{2}}{\tau_{1}} \geq \operatorname{Pr}_{\mathrm{LBGK}}
\end{aligned}
$$

A comment to these formulas is in order: the hierarchy of relaxation times $\left(\tau_{1} \leq \tau_{2}\right)$ implies a restriction on the range of admissible Prandtl number when a specified quasi-equilibrium is used. In the case where the heat flux is considered as the slow variable, the thermal conductivity (relaxation rate of the heat flux) is proportional to the slow relaxation time, $\kappa \sim \tau_{2}$, while $\mu \sim \tau_{1}$. In the opposite case when the stress tensor is chosen as a slow variable, the dependence is inverted, $\mu \sim \tau_{2}$ and $\kappa \sim \tau_{1}$.

Implementation of the present QE models is a straightforward application of equation (19). In Figure 1, simulation of Couette flow between parallel plates at different temperatures is compared with the analytical solution. Diffusive boundary condition was used [14]. Agreement between simulation and analytical solution is excellent.

\subsection{Binary mixture at a given Schmidt number}

Our second example is the isothermal binary mixture of ideal fluids, $\mathrm{A}$ and $\mathrm{B}$, with particles masses $m_{\mathrm{A}}$ and $m_{\mathrm{B}}$. The locally conserved variables $M$ are the densities of the components, $\rho_{\mathrm{A}, \mathrm{B}}$, and the momentum of the mixture $\boldsymbol{j}=\boldsymbol{j}_{\mathrm{A}}+\boldsymbol{j}_{\mathrm{B}}$, where $\boldsymbol{j}_{\mathrm{A}, \mathrm{B}}$ are the momenta of the

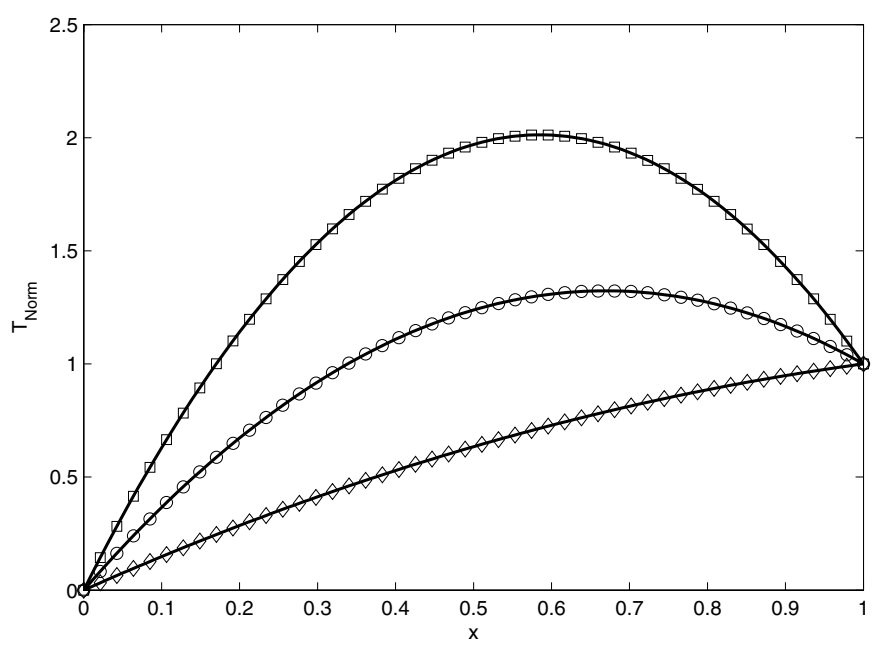

Fig. 1. Steady state temperature profile in Couette flow between parallel plates moving with a relative velocity $U$, at the temperature difference $\delta T$. Lower curve: QELBM (23) with $\operatorname{Pr}=0.710$ (air); middle curve: LBGK model with $\operatorname{Pr}=4$; upper curve: QELBM (24) with $\operatorname{Pr}=8$. Symbol: simulation; line: analytical solution. The Eckert number, Ec $=\left(U^{2}\right) /\left(C_{p} \delta T\right)$ is 1.5 .

components. The efficiency of diffusion mixing versus viscous dissipation of momentum in various fluids is characterized by the Schmidt number, $\mathrm{Sc}=\mu /\left(\rho D_{\mathrm{AB}}\right)$, where $\mu$ is the viscosity coefficient, $D_{\mathrm{AB}}$ is the binary diffusion coefficient, and $\rho=\rho_{\mathrm{A}}+\rho_{\mathrm{B}}$. Let us further introduce the molar fractions $X_{\mathrm{A}, \mathrm{B}}=n_{\mathrm{A}, \mathrm{B}} /\left(n_{\mathrm{A}}+n_{\mathrm{B}}\right)$ where $n_{\mathrm{A}, \mathrm{B}}=\rho_{\mathrm{A}, \mathrm{B}} / m_{\mathrm{A}, \mathrm{B}}$ is the number density of components, and the reference Schmidt number, $\tilde{\mathrm{Sc}}=m_{\mathrm{AB}} /\left(\rho X_{\mathrm{A}} X_{\mathrm{B}}\right)$, with $m_{\mathrm{AB}}=\left(\rho_{\mathrm{A}} \rho_{\mathrm{B}}\right) /\left(\rho_{\mathrm{A}}+\rho_{\mathrm{B}}\right)$ the reduced mass density. We shall now consider a pair of quasi-equilibrium models with two different quasi-equilibria which cover the entire range of Schmidt number, Sc $\lessgtr \tilde{\text { Sc }}$. For concreteness, we use the standard isothermal $D 2 Q 9$ model. For the singlecomponent case, the equilibrium $f_{i}^{\text {eq }}(\rho, \boldsymbol{j})$ to second order in momentum is obtained by setting $p / \rho=T_{0}=1 / 3$ in equation (22). The equilibrium of the mixture is then described by the populations $f_{\mathrm{A}, \mathrm{B} i}^{\mathrm{eq}}=f_{i}^{\mathrm{eq}}\left(\rho_{\mathrm{A}, \mathrm{B}}, \boldsymbol{j}\right)$.

The case $\mathrm{Sc}<\tilde{\mathrm{Sc}}$ has been already considered recently $[11,12]$, and is mentioned here for the sake of completeness. In this case we consider the momenta of the components, $\boldsymbol{j}_{\mathrm{A}, \mathrm{B}}$ as the quasi-conserved variables $N$, and the corresponding quasi-equilibrium is immediately read off the equilibrium of the single-component fluid; to second order in momentum it is $f_{\mathrm{A}, \mathrm{B} i}^{*}\left(\rho_{\mathrm{A}, \mathrm{B}}, \boldsymbol{j}_{\mathrm{A}, \mathrm{B}}\right)=$ $f_{i}^{\text {eq }}\left(\rho_{\mathrm{A}, \mathrm{B}}, \boldsymbol{j}_{\mathrm{A}, \mathrm{B}}\right)$. The continuous $\mathrm{QE}$ model recovers equations of the isothermal hydrodynamics that is, the advection-diffusion equations for the densities $\rho_{\mathrm{A}, \mathrm{B}}$ and the Navier-Stokes equation for the momentum $j$ with $\mu=\tau_{1} p_{0}, D_{\mathrm{AB}}=\tau_{2} p_{0} \frac{X_{\mathrm{A}} X_{\mathrm{B}}}{m_{\mathrm{B}}}$, where $p_{0}=T_{0}\left(n_{\mathrm{A}}+n_{\mathrm{B}}\right)$ is the pressure at the reference temperature $T_{0}$. This model recovers $\mathrm{Sc}=\left(\tau_{1} / \tau_{2}\right) \tilde{\mathrm{Sc}}$, and according to the 


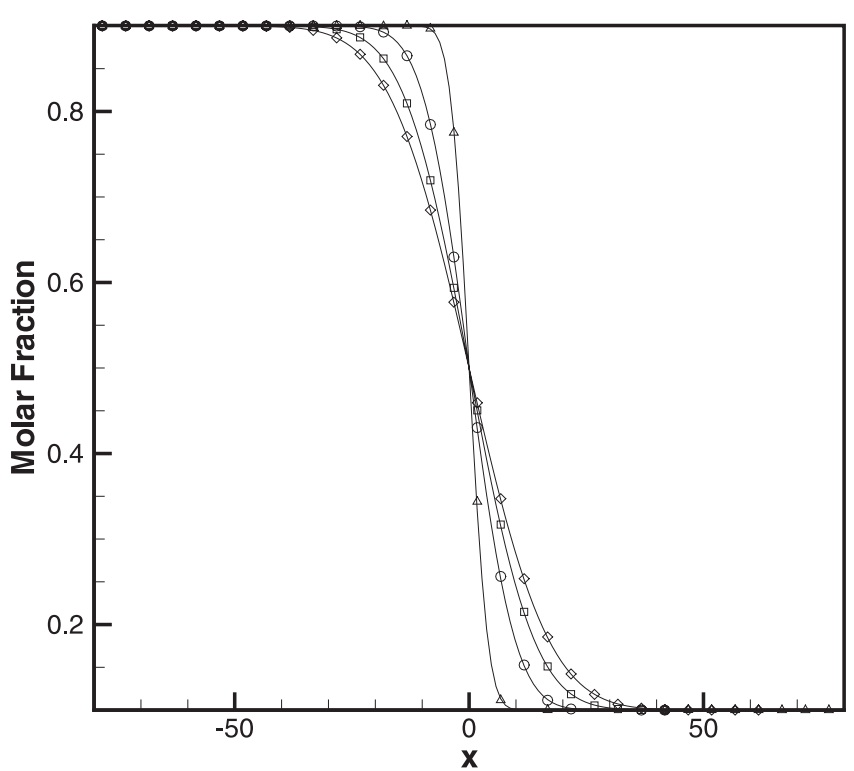

Fig. 2. Binary diffusion for the case $\mathrm{Sc}>\tilde{\mathrm{Sc}}$ and $m_{B} / m_{A}=$ 500 at different time steps $t$. Symbol: simulation; line: analytical solution. Triangles: $t=500$; circles: $t=3000$; squares: $t=6000$; diamonds: $t=9000$.

hierarchy of the relaxation times (7), pertains to the fluids with $\mathrm{Sc} \leq \tilde{\mathrm{Sc}}$.

In the opposite case, $\mathrm{Sc}>\tilde{\mathrm{Sc}}$, we choose the stress tensors of the components, $\boldsymbol{\Theta}_{\mathrm{A}, \mathrm{B}}$, as the quasi-conserved fields $N$ (see Eq. (24)). Corresponding quasi-equilibria, $f_{\mathrm{A}, \mathrm{B} i}^{*}\left(\rho_{\mathrm{A}, \mathrm{B}}, \boldsymbol{j}, \boldsymbol{\Theta}_{\mathrm{A}, \mathrm{B}}\right)$, are constructed using the triangle entropy method in the same way as the case $\operatorname{Pr}>\operatorname{Pr}_{\text {LBGK }}$.

The lattice Boltzmann implementation is based on (19) and an interpolation step, as explained in $[11,12]$. In Figure 2, we present a simulation of diffusion of two fluids with a high mass ratio $m_{\mathrm{B}}=500 \mathrm{~m}_{\mathrm{A}}$ in a setup where initially a half-space is filled with the $90-10 \%$ mixture and the other half-space - with the 10-90\% mixture. Agreement with the analytical solution is excellent.

\section{Conclusion}

In conclusion, we suggested a systematic, physically transparent and realizable approach to constructing lattice Boltzmann models for hydrodynamic systems. All the models considered herein require only the choice of the quasi-equilibria appropriate to the physical context of the problem. Following the same pattern, it is straightforward to construct kinetic models for bulk viscosity and chemical reactions.

\section{References}

1. S. Succi, The Lattice Boltzmann Equation For Fluid Dynamics and Beyond (Oxford University Press, Oxford, 2001)

2. A.N. Gorban, I.V. Karlin, Physica A 206, 401 (1994)

3. C.D. Levermore, J. Stat. Phys. 83, 1021 (1996)

4. I.V. Karlin, A. Ferrante, H.C. Öttinger, Europhys. Lett. 47, 182 (1999)

5. S. Ansumai, I.V. Karlin, H.C. Öttinger, Europhys. Lett. 63, 798 (2003)

6. S. Ansumali, I.V. Karlin, Phys. Rev. Lett. 95, 260605 (2005)

7. S.S. Chikatamarla, S. Ansumali, I.V. Karlin, Phys. Rev. Lett. 97, 010201 (2006)

8. S.S. Chikatamarla, I.V. Karlin, Phys. Rev. Lett. 97, $190601(2006)$

9. A.N. Gorban, I.V. Karlin, Physica A 360, 325 (2006)

10. X. He, S. Chen, G.D. Doolen, J. Comput. Phys. 146, 282 (1998)

11. S. Arcidiacono, S. Ansumali, I.V. Karlin, J. Mantzaras, K.B. Boulouchos, Mathematics and Computers in Simulation 72, 79 (2006)

12. S. Arcidiacono, J. Mantzaras, S. Ansumali, I.V. Karlin, C. Frouzakis, K.B. Boulouchos, Phys. Rev. E 74, 056707 (2006)

13. N.I. Prasianakis, S.S. Chikatamarla, I.V. Karlin, S. Ansumali, K.B. Boulouchos, Mathematics and Computers in Simulation 72, 179 (2006)

14. S. Ansumali, I.V. Karlin, Phys. Rev. E 66, 026311 (2002) 